\title{
LINE STRUCTURES IN THE X-RAY SPECTRA OF CYGNUS X-2 OBSERVED WITH EXOSAT
}

\author{
P. E. Freeman ${ }^{1}$, S. M. Kahn ${ }^{1}$, L. Chiappetti ${ }^{2}$, E. G. Tanzi ${ }^{2}$, A. Ciapi ${ }^{3}$, L. Maraschi ${ }^{3}$, \\ A. Treves ${ }^{3}$, E. G. Branduardi-Raymont, E. N. Ercan \\ ${ }^{1}$ University of Califomia, Berkeley, CA, USA \\ 2 Institute of Cosmic Physics - CNR, Milan, ITA \\ ${ }^{3}$ University of Milan, Milan, ITA \\ ${ }_{5}^{4}$ Mullard Space Science Laboratory, London, GBR \\ ${ }^{5}$ Bogazici University, Bebek, Istanbul, TUR
}

\begin{abstract}
Cygnus X-2 was observed with EXOSAT at five phases of a single orbital cycle in September of 1983. We will summarize the results of spectral fits of the LE + ME (Argon) data in terms of a superposition of thermal bremstrahlung and blackbody components. During the first observation a grating spectrum was obtained and this is described in some detail. The GSPC data are used to investigate the presence of iron features, and their behavior during dips.
\end{abstract}

\section{INTRODUCTION}

Cygnus X-2 was observed by EXOSAT in September 1983, at five equispaced phases of a single $9.8 \mathrm{~d}$ orbital cycle. The aim of the observations was to obtain spectral information in the .1 to $15 \mathrm{KeV}$ band. All the instrumentation onboard the satellite was used toward this end. These included: 1) a medium energy array of proportional counters (ME); 2) a Gas Scintillation Proportional Counter (GSPC); 3) a grazing incidence low energy telescope (LE2), focused on a Channel Multiplier Array and with interposed filters; and 4) a second telescope (LE1) used in conjunction with a 1000 line/mm transmission grating. The grating was used only for the first observation, after which the insertion mechanism jammed.

In a previous paper, light curves and a spectral fit deduced from the ME data were reported (Chiappetti et al. 1987). After the discovery of Quasi-Periodic Oscillations from Cyg X-2 (Hasinger et al. 1986), the short term variability was also reanalyzed (Stella et al. 1986, Chiappetti et al. 1987).

In this paper, we start by giving spectral fits derived from the combination of ME and LE2 data (Section 2), and then concentrate on the line spectra. In Section 3 we report the analysis of GSPC data, while in Section 4 we discuss the analysis of the grating spectrum.

\section{X-RAY CONTINUUM}

During each observation, the LE2 count rates were measured with four filters- pure polypropylene (PPL), parylene coated with aluminum (Al/Par), a $3000 \AA$ Lexan filter, and a boron filter. This measurement was combined with the output of the ME Ar counter and fitted with a standard $\chi^{2}$-square minimization procedure. We report the result of a fit with a superposition of thermal bremsstrahlung and blackbody components, plus interstellar absorption modeled after Morrison and McCammon (1984). Results of the fit are reported in Table 1. 
Following the convention introduced by Vrtilek et al. (1986), during observations one, two, four, and five the source appeared to be in a high state (L2-10 keV $\left.>9 \times 10^{37} \mathrm{erg} / \mathrm{s}\right)$, while during observation three the source was in low state $\left(\mathrm{L}<7 \times 10^{37} \mathrm{erg} / \mathrm{s}\right)$. The fits give parameters comparable to those obtained with the SSS- Einstein experiment (.5 to $4.5 \mathrm{keV}$; Vrtilek et al.). The differences are attributable to the intrinsic variation of the source and the different energy range of the observations.

\section{GSPC OBSERVATIONS OF CYG X-2}

The EXOSAT GSPC is coaligned with the ME array of proportional counters. The GSPC offers the advantage of higher energy resolution (about 10 percent FWHM at $6 \mathrm{keV}$ ), which allows the detection and the detailed study of broad spectral features which cannot be resolved with the ME.

We accumulated GSPC spectra of Cyg X-2 over the energy range 2 to $15 \mathrm{keV}$ for the time intervals covered by the ME observations. The spectra were then fitted with a two-component exponential plus blackbody model similiar to that applied to the ME data; the low-energy column density was held constant at the value determined from the combined LE2 and ME data (see Section 2), since the GSPC is insensitive to densities lower than $10^{22} \mathrm{~cm}^{-2}$. The GSPC best-fit parameters for the $\mathrm{X}$-ray continuum are consistent with those derived from the ME data for each observation. Inclusion of an Fe line at $6.7 \mathrm{keV}$ improves the quality of the fits to the GSPC spectra substantially in the case of observations 1 and 3, and marginally for observations 4 and 5 (quality is determined by $\chi^{2}$ values: e.g. in observation 1 , the inclusion of the Fe line decreases the reduced $\chi^{2}$ value from 1.50 to 1.12 for 203 and 201 degrees of freedom respectively).

Table 2 lists the Fe line energy and equivalent width (EW), along with the 90 percent confidence errors for each of the five observations.

For observations 1 and 2 the parameters listed in Table 2 correspond to time intervals outside the dips. We have investigated the behavior of the Fe line during the dips: the best fits to the GSPC spectra accumulated during the dip intervals do not require inclusion of an Fe line, with a 90 percent confidence upper limit to the EW of 41 and $33 \mathrm{eV}$ respectively. Although these upper limits are not very stringent when compared with the EW measured in observations 3 to 5 , they are significantly different from the values measured in observations 1 and 2 outside of the dips (60 and $55 \mathrm{eV}$ ), and indicate a change in the behavior of the source during the dips.

TABLE 1

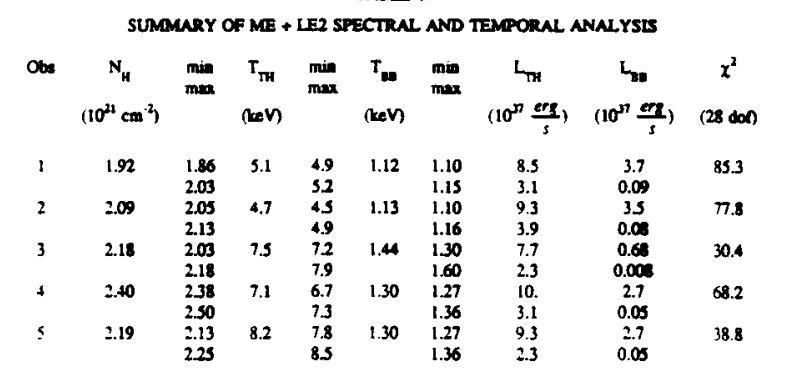

For the luminosition, the opper figue reprosente the value in the 1 to $16 \mathrm{keV}$ bend, while the bourom igure show the value if the 0.2 if $1 \mathrm{beV}$ bed.

TARE 2

OBSERVATIONS OF THE DISCRETE RON FEATURE (GSPC)

\begin{tabular}{|c|c|c|c|c|}
\hline Observation & Phave & Fe line energy (kev) & Fe line EW (eV) & Comanent \\
\hline 1 & 0.96 & $6,67 \pm .07$ & $60 \pm 8$ & dip duries oberming \\
\hline $\begin{array}{l}2 \\
3\end{array}$ & $\begin{array}{l}0.05 \\
0.33\end{array}$ & $\begin{array}{l}6.67 \pm .10 \\
686 \pm .14\end{array}$ & $34 \pm 12$ & \\
\hline 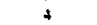 & 0.53 & $6.69 \pm .20$ & $14 \pm 9$ & \\
\hline 5 & 0.75 & $6.57 \pm .23$ & $18 \pm 8$ & \\
\hline
\end{tabular}




\section{GRATING OBSERVATION OF CYG X-2}

The 1000-line Objective Grating Spectrometer (OGS) was used during the first observation, in conjunction with the LE1 telescope and a Channel Multiplier Array (CMA).

The OGS spectrum was fit using a standard $\chi^{2}$-minimization procedure, with parameter constraints introduced by the results of the ME + LE2 data fit. The wider band of this latter spectrum serves to make it more sensitive to temperature and normalization, so these numbers were held constant. In the case of the normalization, an overall fudge factor was introduced, to account for uncertainties in relative effective area calibration between the various instruments. This factor was within ten percent of unity, consistent with the expected uncertainty. Since the grating data are sensitive to absorption, the column density and the abundances in the absorbing medium were allowed to float freely.

Inclusion of all data in the fitting process led to an inability to derive continuum parameters consistent with the ME + LE2 results. Refering to the residuals of the fit between 10 and $19 \AA$, in Figure 2, we note a discrete excess of emission. If this region of the spectrum is excluded, then the continuum model deduced from the ME + LE2 data can also be fitted to the remaining OGS data. The excess is most likely associated with a complex of L-shell emission lines from several multiply-ionized species of iron. The structure of this emission complex is similiar to that found by Vrtilek et al. for Cyg X-2 using the Einstein OGS, though the lower resolution of the EXOSAT OGS precludes definite line identifications in our case (see Table 3). The unknown discrete feature found by Vrtilek et al. at $19.7 \AA$ does not appear in the EXOSAT spectrum.

Also evident in the spectrum is oxygen K-shell absorption edge at $23.3 \AA$. Our spectral fits suggest an abundance 30 percent lower than the cosmic value given by Morrison and McCammon. The abundances of nitrogen and carbon were found to be normal, although the constraints are much less restrictive for these elements.

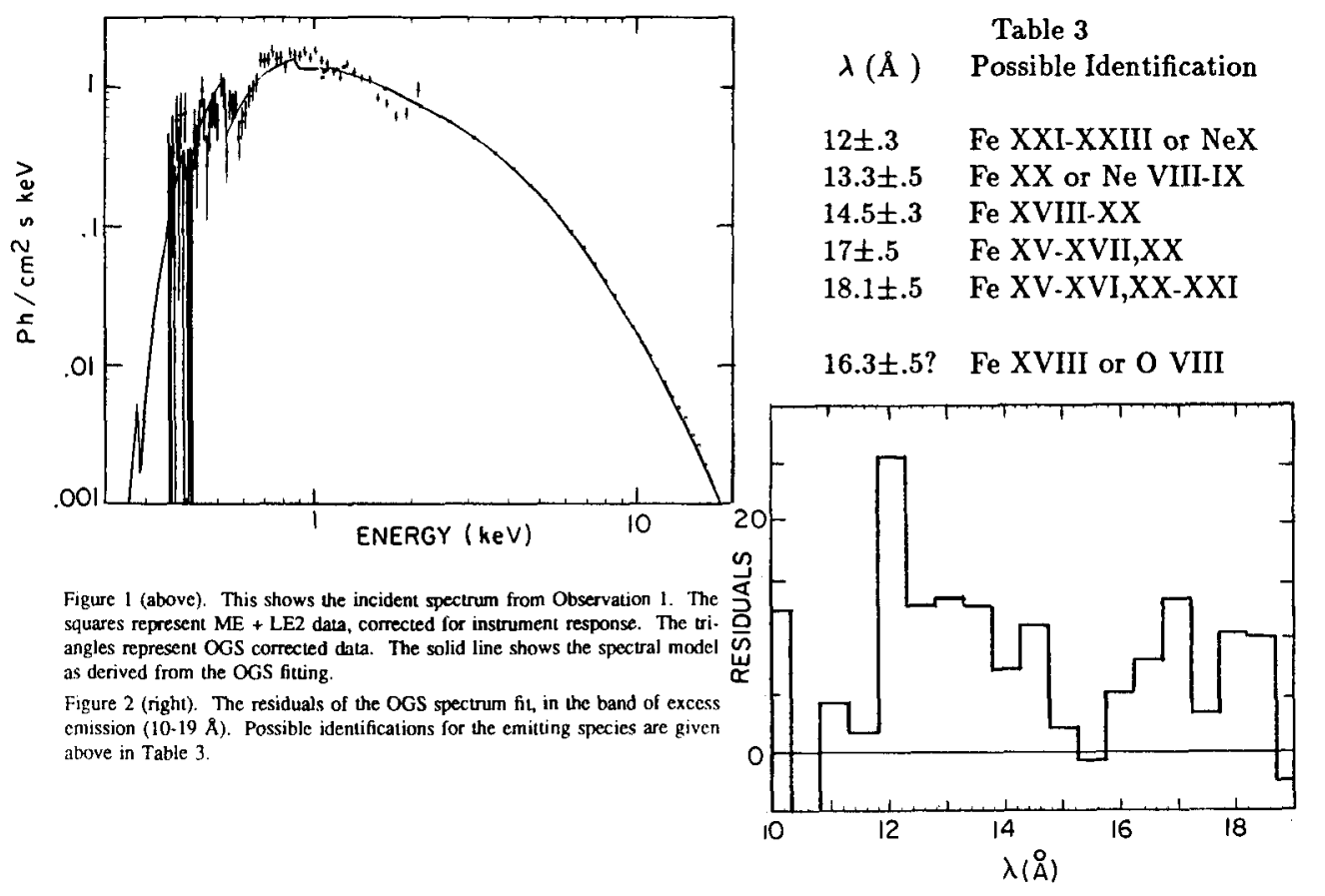




\section{DISCUSSION}

Our observations of Cygnus X-2 using a variety of instruments and covering a wide energy band yield results which are encouragingly consistent with previous studies of this source. In particular, we find that the continuum is well-described by a model involving a superposition of thermal bremsstrahlung and blackbody components.

The GSPC spectral fits explicitly require the presence of a resolved line feature at $6.7 \mathrm{keV}$ which may be attributed to helium-like Fe K-shell emission broadened by multiple Compton scattering in an optically thick emitting region. The derived equivalent width of this feature is similar to that detected for other low mass X-ray binaries. Our data suggest that the equivalent width may anticorrelate with the appearance of "dips" in the X-ray light curve. In the conventional interpretation, these dips are associated with obscuration of the central emitting regions by "bulges" in the accretion flow at the edge of the disk. If this is correct, then the observed decrease in the line equivalent width during dips must indicate that the $\mathrm{Fe} \mathrm{K}$ emitting region is more compact than the continuum emitting region. Presumeably then, the continuum arises in an extended accretion disk corona, whereas the line is produced in a cooler region closer to the compact object. It will be interesting to see if this phenomenon is observed for other sources as well.

The appearance of discrete excesses in the soft X-ray spectrum in the range $10-15 \AA$ confirms the detections of similar features for Cyg X-2 and other sources using the Einstein objective grating spectrometer. These excesses are most likely associated with a complex of $\mathrm{Fe}$ $\mathrm{L}$-shell emission lines produced in a moderate density plasma $\left(\mathrm{n}-10^{11} \mathrm{~cm}^{-3}\right.$ ) located near the edge of the accreting flow. Such material will be photoionized by the strong continuum $\mathrm{X}$-ray flux from the central source. The lines are mostly excited by radiative cascades following recombination. This process is quite complex so that modest changes in the photoionizing flux or in the geometry and density of the accreting flow can produce qualitative variations in the details of the discrete spectral structure. The observed EXOSAT features are similar but not identical to those observed with Einstein. Eventually, detailed comparisons of the variations in these features may yield interesting constraints, however, at present the limited spectral resolution of the grating spectrometer precludes a more sophisticated analysis.

This work was supported in part by a grant from NASA in connection with the Space Astrophysics Data Analysis Program.

\section{REFERENCES}

Chiappetti, L., Maraschi, L., Tanzi, E.G., and Treves, A. 1983, Ap. J., 265, 354.

Chiappetti, L., Ciapi, A.L., Maraschi, L., Stella, L., Tanzi, E.G., and Treves, A. 1987, Ast. and Sp. Sci., 131, 691.

Hasinger, G., Langmeier, A., Sztajno, M., Trumper, J., Lewin, W.H.G., and White, N.E. 1986, Nature, 319, 469.

Hirano, T., Hayakawa, S., Nagase, F., and Tawara, Y. 1986, Ast. and Sp. Sci., 119, 77.

Stella, L., Chiappetti, L., Ciapi, A.L., Maraschi, L., Tanzi, E.G., Treves, A., "Quasi-Periodic Oscillations in Cyg X-2" in Proceedings of the Fourth Marcel Grossman Meeting on General Relativity, Rome, June 1985, Ed. Ruffini, R.

Vrtilek, S.D., Kahn, S.M., Grindlay, J.E., Helfand, D.J., and Seward, F.D. 1986, Ap. J., 307, 698.

White, N.E., Peacock, A., Hasinger, G., Mason, K.O., Manzo, G., Taylor, B.G., and BranduardiRaymont, G. 1986, Mon. Not. R. Astr. Soc., 218, 129. 